PROCEEDINGS OF THE

AMERICAN MATHEMATICAL SOCIETY

Volume 128, Number 12, Pages 3541-3545

S 0002-9939(00)05780-4

Article electronically published on May 18, 2000

\title{
STOCHASTIC STABILITY OF GENERALIZED SRB MEASURES OF AXIOM A BASIC SETS
}

\author{
LIU PEI-DONG AND ZHENG HONG-WEN
}

(Communicated by Michael Handel)

\begin{abstract}
In this note we prove that the generalized SRB measure of an Axiom A basic set is stable under random diffeomorphisms type perturbations.
\end{abstract}

In her paper [7], Young proved that the SRB (Sinai-Ruelle-Bowen) measure of a hyperbolic attractor of a diffeomorphism is stable under i.i.d. (independent and identically distributed) random diffeomorphisms type perturbations. In the case of an Axiom A basic set of a diffeomorphism, the generalized SRB measure was proved in Liu [5] to persist under more general, i.e., stationary, random diffeomorphisms type perturbations, by employing the Markov partition technique and the thermodynamic formalism of random subshifts of finite type (see 5 for the related references). In this note we prove that the generalized SRB measure of an Axiom A basic set is stable with respect to such perturbations, i.e., the random generalized SRB measure converges weakly to the deterministic one when the scale of the perturbation tends to zero.

In this paper, $M$ will always be a Riemannian manifold without boundary, $O$ an open subset of $M$ with compact closure and $f: O \rightarrow M$ a $C^{r}(r \geq 1)$ diffeomorphism to the image $f O$. Assume that $\Lambda_{0} \subset O$ is an Axiom A basic set of $f$, i.e., it is a transitive locally maximal hyperbolic set of $f$ (see [5] for the present and later various related notions).

Let $C^{r}(O, M)(r \geq 1)$ be the space of all $C^{r}$ maps from $O$ to $M$, equipped with the $C^{r}$ compact-open topology. It is well known that $C^{r}(O, M)$ can be metrized to a Polish space. We will fix such a metric on $C^{r}(O, M)$.

Let $\mathcal{U}(f)$ be an open neighborhood of $f$ in $C^{r}(O, M)$ and consider a random variable taking values in $\mathcal{U}(f)$,

$$
F:(\Omega, \mathcal{F}, P) \rightarrow \mathcal{U}(f),
$$

where $(\Omega, \mathcal{F}, P)$ is a complete probability space such that $\mathcal{F}$ is countably generated and on which there is an invertible ergodic measure-preserving transformation $\theta$ that represents the time evolution of the underlying noise system. Put for each

Received by the editors January 28, 1999.

2000 Mathematics Subject Classification. Primary 37D20.

Key words and phrases. Axiom A basic set, generalized SRB measure, random dynamical system.

The first author was supported by the NSPCP and a fund from the NECC.

The second author was supported by the National Natural Science Foundation of China.

(C)2000 American Mathematical Society 
$\omega \in \Omega$

$$
F(n, \omega)= \begin{cases}F\left(\theta^{n-1} \omega\right) \circ \cdots \circ F(\omega) & \text { if } n>0, \\ \text { id } & \text { if } n=0, \\ F\left(\theta^{n} \omega\right)^{-1} \circ \cdots \circ F\left(\theta^{-1} \omega\right)^{-1} & \text { if } n<0,\end{cases}
$$

defined wherever they make sense. Such an RDS (random dynamical system) generated by $F$ will be considered as a random perturbation of $\left(f, \Lambda_{0}\right)$. We will also denote this RDS by $F$ and write $F(1, \omega)$ simply as $F(\omega)$. This general model of random perturbation was proposed by Bogenschütz ([2] and [3] $)$. The reader is also referred to Arnold [1] for a comprehensive theory of RDS.

The following result on structural stability of $\left(f, \Lambda_{0}\right)$ with respect to sample perturbations follows from Theorem 1.1 and Proposition 1.5 of Liu [5].

Proposition 1. Let $r=1$. Then there exist a number $\varepsilon_{0}>0$ and an open neighborhood $\mathcal{V}(f)$ of $f$ in $C^{1}(O, M)$ such that for any $R D S$ generated by a random variable $F:(\Omega, \mathcal{F}, P) \rightarrow \mathcal{V}(f)$ the following hold true:

(1) For each $\omega \in \Omega$ and any $x \in \Lambda_{0}$ there exists a unique orbit $\left\{x_{\omega}(n)\right\}_{n \in \mathbf{Z}}$ of $F(\cdot, \omega)$ such that

$$
d\left(f^{n} x, x_{\omega}(n)\right) \leq \varepsilon_{0}
$$

for all $n \in \mathbf{Z}$. For any given $0<\varepsilon \leq \varepsilon_{0}$ one can shrink the $\mathcal{V}(f)$ given above so that (1) holds true with $\varepsilon_{0}$ being replaced by $\varepsilon$.

(2) Let $\omega \in \Omega$. Then $\Lambda_{\omega}:=\left\{x_{\omega}(0): x \in \Lambda_{0}\right\}$ is compact and depends measurably on $\omega$, and

$$
h_{\omega}: \Lambda_{0} \rightarrow \Lambda_{\omega}, x \mapsto x_{\omega}(0)
$$

is a homeomorphism that also depends measurably on $\omega$. Moreover, $F(\omega): \Lambda_{\omega} \rightarrow$ $\Lambda_{\theta \omega}$ is a homeomorphism and the following diagram is commutative:

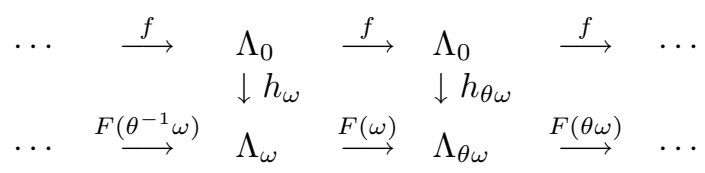

(3) Put

$$
\Lambda=\bigcup_{\omega \in \Omega}\{\omega\} \times \Lambda_{\omega}
$$

and let $E_{\Lambda}$ be the pull-back of $T M$ by means of the projection $p_{2}: \Lambda \rightarrow M,(\omega, x) \mapsto$ $x$. Then there is a measurable splitting $E_{\Lambda}=E_{\Lambda}^{s} \oplus E_{\Lambda}^{u}$ together with a number $0<\lambda<1$ such that for each $(\omega, x) \in \Lambda$

$$
T_{x} F(\omega) E_{(\omega, x)}^{s}=E_{\Phi(\omega, x)}^{s}, \quad T_{x} F(\omega) E_{(\omega, x)}^{u}=E_{\Phi(\omega, x)}^{u}
$$

and

$$
\begin{gathered}
\left|T_{x} F(\omega) \xi\right| \leq \lambda|\xi| \text { for } \xi \in E_{(\omega, x)}^{s}, \\
\left|T_{x} F(\omega) \eta\right| \geq \lambda^{-1}|\eta| \text { for } \eta \in E_{(\omega, x)}^{u},
\end{gathered}
$$

where

$$
\Phi: \Lambda \rightarrow \Lambda,(\omega, x) \mapsto(\theta \omega, F(\omega) x)
$$

is the associated skew-product transformation on $\Lambda$. 
Let $\Lambda=\bigcup_{\omega \in \Omega}\{\omega\} \times \Lambda_{\omega}$ be as given in Proposition 1. Then

$$
\left\{F(n, \omega): \Lambda_{\omega} \rightarrow \Lambda_{\theta^{n} \omega}, n \in \mathbf{Z}, \omega \in \Omega\right\}
$$

defines a bundle RDS over $(\Omega, \mathcal{F}, P, \theta)$ and we will still denote this RDS by $F$ for convenience of notation.

A family of functions $\varphi=\left\{\varphi_{\omega} \in C\left(\Lambda_{\omega}\right)\right\}_{\omega \in \Omega}$ is called an equi-Hölder continuous random function on $\Lambda$ if $(\omega, x) \mapsto \varphi_{\omega}(x)$ is measurable on $\Lambda, \int \sup _{x \in \Lambda_{\omega}}\left|\varphi_{\omega}(x)\right| d P(\omega)$ $<+\infty$ and there exist constants $C>0$ and $\alpha>0$ such that for $P$-a.e. $\omega$ one has $\left|\varphi_{\omega}(x)-\varphi_{\omega}(y)\right| \leq C d(x, y)^{\alpha}$ for any $x, y \in \Lambda_{\omega}$. The proof of the following result is similar to those of the analogous results in Liu $[\underline{5}$. (As said above, see $[5]$ for the various related concepts.)

Proposition 2. (1) Let $r=1$. The neighborhood $\mathcal{V}(f)$ in Proposition 1 can be taken so that, if $F$ is the bundle $R D S$ on $\Lambda$ as introduced above, then for any equiHölder continuous random function $\varphi$ on $\Lambda$ there exists a unique equilibrium state, written $\tilde{\mu}_{\varphi}$, of $\varphi$ with respect to $F$.

(2) Let $r=2$. Then $\mathcal{V}(f)$ can be taken to be an open neighborhood of $f$ in $C^{2}(O, M)$ such that, if $F$ is the bundle $R D S$ on $\Lambda$ as introduced above and if one defines $\varphi_{\omega}^{(u)}: \Lambda_{\omega} \rightarrow \mathbf{R}$ by

$$
\varphi_{\omega}^{(u)}(x)=-\log \mid \operatorname{det}\left(\left.T_{x} F(\omega)\right|_{\left.E_{(\omega, x)}^{u}\right)}\right),
$$

then $\varphi^{(u)}:=\left\{\varphi_{\omega}^{(u)}\right\}_{\omega \in \Omega}$ is an equi-Hölder continuous random function on $\Lambda$ and hence there exists a unique equilibrium state, written $\tilde{\mu}^{(u)}$, of $\varphi^{(u)}$ with respect to $F . \tilde{\mu}^{(u)}$ satisfies

$$
\pi_{F}\left(\varphi^{(u)}\right)=h_{\tilde{\mu}^{(u)}}(F)-\sum \text { positive Lyapunov exponents of }\left(F, \tilde{\mu}^{(u)}\right),
$$

where $\pi_{F}\left(\varphi^{(u)}\right)$ is the pressure of $\varphi^{(u)}$ with respect to $F$ and $h_{\tilde{\mu}^{(u)}}(F)$ is the entropy of $\left(F, \tilde{\mu}^{(u)}\right)$. This measure is called the generalized SRB measure of the bundle RDS $F$.

In what follows we consider the stability of the generalized SRB measure of $f$ on $\Lambda_{0}$ with respect to small random perturbations as introduced above. Let $r=2$. For $\varepsilon>0$ let $\mathcal{U}_{\varepsilon}(f)$ be the $\varepsilon$-neighborhood of $f$ in $C^{2}(O, M)$ and assume that

$$
F_{\varepsilon}:(\Omega, \mathcal{F}, P) \rightarrow \mathcal{U}_{\varepsilon}(f)
$$

is a random variable. When $\varepsilon$ is sufficiently small, let $F_{\varepsilon}$ be the corresponding bundle RDS on the corresponding set $\Lambda_{\varepsilon}=\bigcup_{\omega \in \Omega}\{\omega\} \times \Lambda_{\varepsilon, \omega}$. Let $h_{\varepsilon, \omega}: \Lambda_{0} \rightarrow$ $\Lambda_{\varepsilon, \omega}, \omega \in \Omega$ be the associated conjugate homeomorphisms and let $\tilde{\mu}_{\varepsilon}^{(u)}$ be the corresponding generalized SRB measure of $F_{\varepsilon}$. Putting $X=\bar{O}$ we denote by $\mathcal{M}_{P}(\Omega \times X)$ the set of measures on $(\Omega \times X, \mathcal{F} \times \mathcal{B}(X))$ which have marginal $P$ on $\Omega$. Let $L^{1}(\Omega, C(X))$ be the set of random functions $\psi=\left\{\psi_{\omega} \in C(X)\right\}_{\omega \in \Omega}$ which are such that $(\omega, x) \mapsto \psi_{\omega}(x)$ is measurable and $\int \sup _{x \in X}\left|\psi_{\omega}(x)\right| d P(\omega)<+\infty$. For $\psi \in L^{1}(\Omega, C(X))$ and $\tilde{\mu} \in \mathcal{M}_{P}(\Omega \times X)$, write

$$
\int \psi d \tilde{\mu}=\iint \psi_{\omega}(x) d \tilde{\mu}_{\omega}(x) d P(\omega)
$$

where $\left\{\tilde{\mu}_{\omega}\right\}$ is the natural decomposition of $\tilde{\mu}$. For $\tilde{\mu}_{n}, \tilde{\mu} \in \mathcal{M}_{P}(\Omega \times X), n=1,2, \cdots$, by saying that $\tilde{\mu}_{n}$ converges weakly to $\tilde{\mu}$ we mean that

$$
\lim _{n \rightarrow+\infty} \int \psi d \tilde{\mu}_{n}=\int \psi d \tilde{\mu}
$$


for all $\psi \in L^{1}(\Omega, C(X))$. Considering each $\tilde{\mu}_{\varepsilon}^{(u)}$ as an element of $\mathcal{M}_{P}(\Omega \times X)$, we state our main result of this note as follows.

Theorem 3. In the sense of weak convergence of measures in $\mathcal{M}_{P}(\Omega \times X)$, one has

$$
\lim _{\varepsilon \rightarrow 0} \tilde{\mu}_{\varepsilon}^{(u)}=P \times \mu_{0}
$$

where $\mu_{0}$ is the generalized $S R B$ measure of $f$ on $\Lambda_{0}$. In particular, the marginal of $\tilde{\mu}_{\varepsilon}^{(u)}$ on $X$ converges weakly in the usual sense to $\mu_{0}$ as $\varepsilon \rightarrow 0$, i.e.,

$$
\lim _{\varepsilon \rightarrow 0} \mu_{\varepsilon}^{(u)}=\mu_{0}
$$

where $\mu_{\varepsilon}^{(u)}(\cdot):=\int\left(\tilde{\mu}_{\varepsilon}^{(u)}\right)_{\omega}(\cdot) d P(\omega)$.

Proof. Since $\mathcal{M}_{P}(\Omega \times X)$ is compact, it suffices to show that, if

$$
\lim _{n \rightarrow+\infty} \tilde{\mu}_{\varepsilon_{n}}^{(u)}=\tilde{\mu}
$$

for $\varepsilon_{n} \rightarrow 0$ as $n \rightarrow+\infty$, then $\tilde{\mu}=P \times \mu_{0}$.

Let $F_{0}$ denote the trivial $\operatorname{RDS}\left\{f: \Lambda_{0} \rightarrow \Lambda_{0}\right\}_{\omega \in \Omega}$ over $(\Omega, \mathcal{F}, P, \theta)$, and define $\varphi_{0}^{(u)}=\left\{\varphi_{0, \omega}^{(u)}: \Lambda_{0} \rightarrow \mathbf{R}\right\}_{\omega \in \Omega}$ by $\varphi_{0, \omega}^{(u)}(x)=-\log \left|\operatorname{det}\left(\left.T_{x} f\right|_{E_{x}^{u}}\right)\right|\left(E_{x}^{u}\right.$ the unstable space of $f$ at $x$ ). Clearly $P \times \mu_{0}$ is the unique equilibrium state of $\varphi_{0}^{(u)}$ with respect to $F_{0}$.

We first show that for the pressure one has

$$
\lim _{\varepsilon \rightarrow 0} \pi_{F_{\varepsilon}}\left(\varphi_{\varepsilon}^{(u)}\right)=\pi_{F_{0}}\left(\varphi_{0}^{(u)}\right) .
$$

Since the bundle RDS's $F_{\varepsilon}$ and $F_{0}$ over $(\Omega, \mathcal{F}, P, \theta)$ are isomorphic via the family of homeomorphisms $h_{\varepsilon}:=\left\{h_{\varepsilon, \omega}: \Lambda_{0} \rightarrow \Lambda_{\varepsilon, \omega}\right\}_{\omega \in \Omega}$, one has

$$
\pi_{F_{\varepsilon}}\left(\varphi_{\varepsilon}^{(u)}\right)=\pi_{F_{0}}\left(\varphi_{\varepsilon}^{(u)} \circ h_{\varepsilon}\right)
$$

and hence

$$
\begin{aligned}
\left|\pi_{F_{\varepsilon}}\left(\varphi_{\varepsilon}^{(u)}\right)-\pi_{F_{0}}\left(\varphi_{0}^{(u)}\right)\right| & =\left|\pi_{F_{0}}\left(\varphi_{\varepsilon}^{(u)} \circ h_{\varepsilon}\right)-\pi_{F_{0}}\left(\varphi_{0}^{(u)}\right)\right| \\
& \leq \int \sup _{x \in \Lambda_{0}}\left|\varphi_{\varepsilon, \omega}^{(u)}\left(h_{\varepsilon, \omega} x\right)-\varphi_{0}^{(u)}(x)\right| d P(\omega) .
\end{aligned}
$$

Take a neighborhood $U$ of $\Lambda_{0}$ such that the hyperbolic splitting $T_{\Lambda_{0}} M=E^{s} \oplus E^{u}$ can be extended to a continuous splitting $T_{U} M=E^{1} \oplus E^{2}$. By Liu and Qian [6] Proposition VII.2.1] one knows that, if $\varepsilon$ is sufficiently small and if $E_{\Lambda_{\varepsilon}}=E_{\Lambda_{\varepsilon}}^{s} \oplus E_{\Lambda_{\varepsilon}}^{u}$ is the splitting given by Proposition 1 corresponding to $F_{\varepsilon}$, then $E_{\Lambda_{\varepsilon}}^{u}=\operatorname{Graph}\left(L_{\varepsilon}\right)$, where $L_{\varepsilon}: E_{\Lambda_{\varepsilon}}^{2} \rightarrow E_{\Lambda_{\varepsilon}}^{1}$ is a fiber-preserving linear map such that $\left|L_{\varepsilon}\right| \leq \delta_{\varepsilon}$ and $\delta_{\varepsilon} \rightarrow 0$ as $\varepsilon \rightarrow 0$ (here $E_{\Lambda_{\varepsilon}}^{i}$ is the obvious pull-back of $E^{i}$ to $\Lambda_{\varepsilon}, i=1,2$, and in what follows we will identify $\left(E_{\Lambda_{\varepsilon}}^{i}\right)_{(\omega, x)}$ with $E_{x}^{i}$ for each $\left.(\omega, x) \in \Lambda_{\varepsilon}\right)$.

Then, writing $x_{\varepsilon, \omega}=h_{\varepsilon, \omega} x$ and $T_{\varepsilon, \omega, x}=T_{x_{\varepsilon, \omega}} F_{\varepsilon}(\omega)$, one has

$$
\begin{aligned}
\varphi_{0}^{(u)}(x)-\varphi_{\varepsilon, \omega}^{(u)}\left(h_{\varepsilon, \omega} x\right) & =\log \frac{\left|\operatorname{det}\left(\left.T_{\varepsilon, \omega, x}\right|_{\left.E_{\left(\omega, x_{\varepsilon, \omega}\right.}^{u}\right)}\right)\right|}{\left|\operatorname{det}\left(\left.T_{x_{\varepsilon, \omega}} f\right|_{E_{x_{\varepsilon, \omega}}^{2}}\right)\right|}+\log \frac{\left|\operatorname{det}\left(\left.T_{x_{\varepsilon, \omega}} f\right|_{E_{x_{\varepsilon}, \omega}^{2}}\right)\right|}{\left|\operatorname{det}\left(\left.T_{x} f\right|_{E_{x}^{2}}\right)\right|} \\
& :=\Delta_{\varepsilon}^{(1)}(\omega, x)+\Delta_{\varepsilon}^{(2)}(\omega, x) .
\end{aligned}
$$

Note that

$$
\Delta_{\varepsilon}^{(1)}(\omega, x)=\log \frac{\left|T_{\varepsilon, \omega, x}\left(e_{1}+L_{\varepsilon} e_{1}\right) \wedge \cdots \wedge T_{\varepsilon, \omega, x}\left(e_{u}+L_{\varepsilon} e_{u}\right)\right|}{\left|\left(e_{1}+L_{\varepsilon} e_{1}\right) \wedge \cdots \wedge\left(e_{u}+L_{\varepsilon} e_{u}\right)\right|\left|T_{x_{\varepsilon, \omega}} f e_{1} \wedge \cdots \wedge T_{x_{\varepsilon, \omega}} f e_{u}\right|}
$$


where $e_{1}, \cdots, e_{u}\left(u=\operatorname{dim} E^{u}\right)$ is an orthonormal basis of $E_{x_{\varepsilon, \omega}}^{2}$. From this expression one can see that

$$
\lim _{\varepsilon \rightarrow 0} \sup _{\omega \in \Omega, x \in \Lambda_{0}}\left|\Delta_{\varepsilon}^{(1)}(\omega, x)\right|=0 .
$$

Similarly for $\Delta_{\varepsilon}^{(2)}(\omega, x)$. Hence one proves (3).

By the Tietz Extension Theorem, one can extend $\varphi_{0, \omega}^{(u)}(x)=-\log \left|\operatorname{det}\left(\left.T_{x} f\right|_{E_{x}^{u}}\right)\right|$ to a continuous function on $X=\bar{O}$. Then it is easy to see that the above reasoning also yields that, if (2) is true, one has

$$
\lim _{n \rightarrow+\infty} \int_{\Lambda_{\varepsilon_{n}}} \varphi_{\varepsilon_{n}}^{(u)} d \tilde{\mu}_{\varepsilon_{n}}^{(u)}=\int_{\Omega \times \Lambda_{0}} \varphi_{0}^{(u)} d \tilde{\mu} .
$$

Then, by arguments in Liu [5, Subsections 2.2 and 2.3] together with Bogenschütz [4, Proposition 3.2.2] (which says that the entropy map of a random subshift of finite type is upper semi-continuous), one can see that (2), (3) and (4) imply that the corresponding Gibbs lifting measures of $\tilde{\mu}_{\varepsilon_{n}}^{(u)}$ to the symbol representations of $F_{\varepsilon_{n}}$ (which are random subshifts of finite type) as constructed in [5] converge to the corresponding Gibbs lifting of $P \times \mu_{0}$ and hence that

$$
\tilde{\mu}=P \times \mu_{0} .
$$

The proof is completed.

\section{ACKNOWLEDGMENTS}

The authors express their sincere thanks to Drs. T. Bogenschütz and G. Ochs for very helpful discussions. They are also very grateful to Professor He Lian-Fa for his warm advice and encouragement.

\section{REFERENCES}

[1] L. Arnold, Random Dynamical Systems, Springer-Verlag, Berlin Heidelberg New York, 1998.

[2] T. Bogenschütz, Private communication.

[3] T. Bogenschütz, Stochastic stability of equilibrium states, Random Comput. Dynam. 4 (1996), 85-98. MR 97c:58085

[4] T. Bogenschütz, Equilibrium states for random dynamical systems, Ph.D. Thesis, Bremen University, 1993.

[5] P.-D. Liu, Random perturbations of Axiom A basic sets, J. Stat. Phys. 90(1/2) (1998), 467-490. MR 99a:58108

[6] P.-D. Liu, M. Qian, Smooth Ergodic Theory of Random Dynamical Systems, Lec. Not. Math. 1606, Springer, 1995. MR 96m:58139

[7] L.-S. Young, Stochastic stability of hyperbolic attractors, Ergod. Theory Dynam. Syst. 6 (1986), 311-319. MR 88a:58160

Department of Mathematics and Institute of Mathematics, Peking University, BeiJing 100871, People's Republic of China

E-mail address: lpd@pku.edu.cn

Department of Mathematics, Hebei Normal University, Shijiazhuang City 050016, Hebei, People's Republic of China 